\title{
A Novel Architecture to Reduce Dispersion in Fiber by Using Pre, Post and Symmetrical DCF Methods
}

\author{
N. Nerkar, M. Kadu and R. Labade \\ Department of Electronics and Telecommunication, AVCOE Sangamner,India \\ \{narendranerkar4@gmail.com ${ }^{1}$, Mahesh.kadu@gmail.com², rplabade@gmail.com ${ }^{3}$ \}
}

\begin{abstract}
Dispersion is one of the basic problems in the optical fiber communication. Chromatic dispersion is one of this type of dispersion. Nowadays we require high data transmission without any substantial loss in a communication system, so optical communication serves as a better choice for high data rate transmission. However it has a dispersion problem. To overcome dispersion in an optical communication system, we proposed a novel type of method to reduce chromatics dispersion in this paper. The proposed method is experimentally implemented and analysed. The eye diagram analysis test confirms achievement of complete reduction of chromatic dispersion in the fiber at long distance communication. Optisystem version 12.0.2 was used as a software tool for experimental validation of proposed method. Optical fiber system implemented in this experiment was designed for bit rate $2.5 \mathrm{Gbps}$ at wavelength $1550 \mathrm{~nm}$.
\end{abstract}

Keyword: Bit Error Rate, Eye diagram, Dispersion.

\section{Introduction}

Nowadays, with high growth of internet a need of a high capacity of network systems is felt. Demand for higher bandwidth and capacity have become much more challenging factor for a service provider. In these conditions optical communication is most favourable medium for delivering data to the user with excellent bandwidth and transmission performance among the existing communication techniques.

An optical transmission system suffers from an inherent problem of dispersion. This is the main concern for a optical communication network [1]. Dispersions of transmitted optical signal cause distortion for both digital as well as analog signal. Dispersion in the fiber is one of the limiting factors to decide how much data can be sent along alive. Single Mode Fiber use in the high-speed fiber communication network suffers from the dispersion type known as chromatic dispersion. The Chromatic dispersion has a wavelength dependant nature.

The Erbium dope fiber amplifier (EDFA), is considered as a better candidate for high data rate transmission. EDFA works on $1550 \mathrm{~nm}$ wavelength, at that wavelength Single Mode Fiber (SMF) dispersion measured is nearly about $15-20 \mathrm{ps} /\left(\mathrm{nm} \cdot \mathrm{km}^{-1}\right)$ [2]. So it shows that dispersion is the main problem of long distance communication. Also when optical network moves from $2.5 \mathrm{Gbps}$ to $10 \mathrm{Gbps}$ and onward the acceptable tolerance of dispersion is drastically reduced. Dispersion is reduced by the factor of 16 when moving $2.5 \mathrm{Gbps}$ to $10 \mathrm{Gbps}$ and by and an additional factor of 16 moving from $10 \mathrm{Gbps}$ to $40 \mathrm{Gbps}$ [3].

\section{Chromatic Dispersion}

Chromatic dispersion depends on frequency [4]. We all know rainbow is the best example of dispersion in nature of chromatic dispersion. In rainbow there is a spatial separation of white colour into a different wavelength of seven colours. However, dispersion also effects in many other circumstances. For example dispersion caused due to the broadening of pulses in optical fiber degrades signal over long distance. Usually we consider that dispersion effects for light waves, but it's also occurs in non-homogeneous medium geometry (e.g. A waveguide and in sound waves.). In single mode fiber (SMF) dispersion has main two component [5]

I. Wavelength dispersion: This occurs because of different refractive index (R.I.) of core and cladding. Short wavelength light travels within core, medium wavelength light spread slightly into the cladding and long wavelength light spread much into the cladding.

B. Iyer, S. Nalbalwar and R. Pawade (Eds.)

ICCASP/ICMMD-2016. Advances in Intelligent Systems Research.

Vol. 137, Pp. 610-616.

(C) 2017- The authors. Published by Atlantis Press

This is an open access article under the CC BY-NC license (http://creativecommons.org/licens)es/by-nc/4). 
II. Material Dispersion : It is come from frequency dependant respond of material to wave. This cause propagation delay in wavelength relative to other.

As chromatic dispersion is broadening of the signal in an optical cable whiles signal are transmitted. For dispersion, a propagation constant $(\beta)$ is consider. It determines the speed at which pulse energy propagate in fiber. For propagation of electromagnetic wave (EMW) in a homogeneous and monochromatic medium[5].

$$
\text { Propagation constant }(\beta)=\frac{w n}{c}=\frac{2 \pi n}{\lambda},
$$

Where, $n=$ Refractive index of homogeneous medium.

$$
\text { Wave no. }(\mathrm{K})=\frac{2 \pi}{\lambda}
$$

Unit of propagation constant is radian/length.

\subsection{Group-velocity dispersion:}

It is the cause to pulse the signal to spread in optical fiber cable and degrading signal over long distance. As cancellation between group velocity dispersion and nonlinear effect leads to soliton waves. It is second order derivatives of $\beta$ w.r.t. optical frequency.

$$
\beta_{2}=d^{2} \beta / \mathrm{d} \omega^{2}
$$

$\beta_{2}=\mathrm{It}$ is the key parameter governing the revolution of the pulse shape.

In absence of chromatic dispersion $\beta_{2}=0$

When $\beta_{2}>0$ then CD is normal

When $\beta_{2}<0$ then $\mathrm{CD}$ is anomalous.

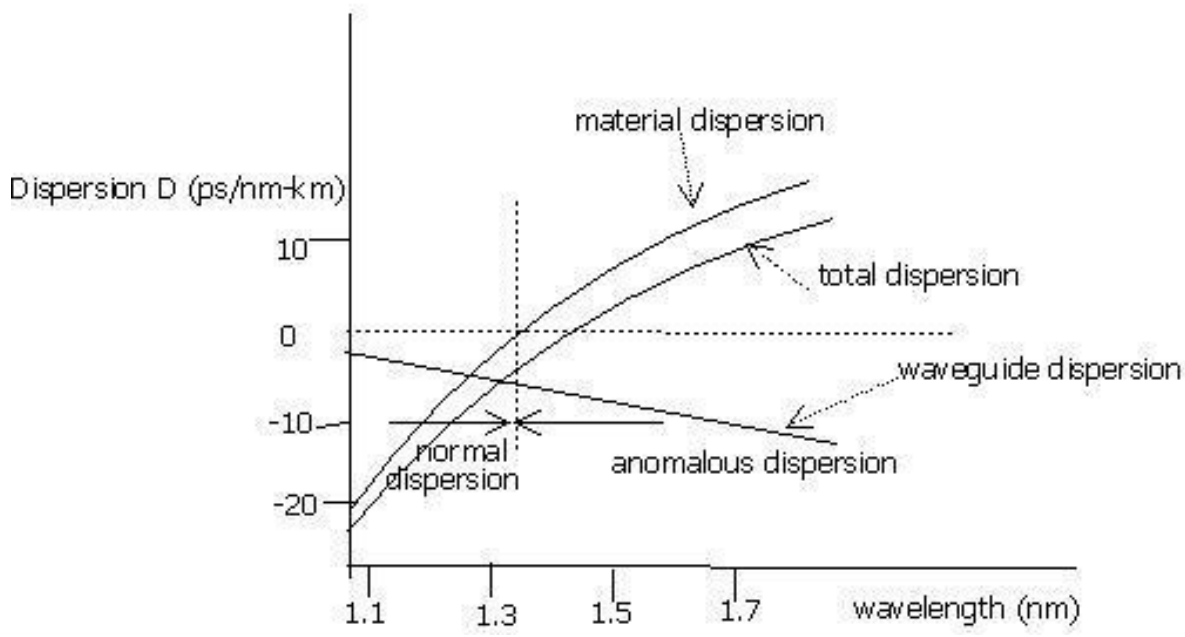

Figure 1: Property of dispersion in SMF [2].

The following are the parameter for a dispersion which considers for transmission.

The chromatic dispersion coefficient is a primary parameter for data loss [5]. To determine CD following equation are consider.

$$
\mathrm{D}(\lambda)=\frac{\mathrm{dt}(\lambda)}{\mathrm{d}(\lambda)}, \frac{\mathrm{ps}}{\mathrm{nm} * \mathrm{~km}}
$$

As $\mathrm{D}(\lambda)=$ Chromatic dispersion coefficient ; $\mathrm{dt}_{\mathrm{g}}(\lambda)=$ Delay time of chromatic dispersion, the value of CD is numerically equivalent to the Gaussian value of pulse (in ps.) an initial spectral half width of $1 \mathrm{~nm}[6]$. 


\section{Dispersion Compensation Technologies}

To improve the transmission performance which effected by dispersion, A no. of technologies is proposed to compensate dispersion. Among them, various techniques are classified as: Dispersion compensating fiber (DCF), Chirped fiber Bragg grading, higher order mode fiber (HOM). In which the DCF mean dispersion compensating fiber are discussed below. A DCF techniques introduced in 1980 [1], but until after introduction of an optical amplifier. A DCF is widely spread, attention and study. For $2^{\text {nd }}$ order and $3^{\text {rd }}$ order dispersion value for SMF is negative. So insulting DCF, the average value is being closed to zero. For wireless C-band, a dispersion compensating fiber has negative dispersion value about 70 to $90 \mathrm{ps} / \mathrm{nm}$. km, Use positive dispersion of transmission fiber [3]. For a relative position of DCF and fiber we introduced following method of compensation and of dispersion: Pre-Compensation, Post-Compensation, and Symmetrical/mixed Compensation method. In Pre-Compensation method is achieved by placing the DCF before a conventional single mode fiber or transmitter of the system. As in the Post-Compensation method, it is the reverse of Pre-Compensation means, here DCF is placed after SMF. In Symmetrical or mixed scheme, we consist here Pre and Post Compensation. The final result will be higher and more accurate than Pre and Post Compensation methods.

\section{Methodology}

Here for analysis purpose we can use optiwaves Optisystem version 12.0 software for simulation and designing of system. Optisystem is a powerful, innovative software tool for optical system designing. In figure 2 , we describe implementation of optical fiber system with single mode fiber. We can seen in the figure DCF is used after SMF means it Post-Compensated fiber method. In between SMF and DCF we use Optical time domain visualiser (OTDR) for measuring spectrum of Bit Error Rate (BER) and threshold value. In figure 3, three compensation scheme shows. In table 1, shows parameter with use in simulation process. In the transmission section we use Mach-Zehnder modulator, NRZ drivers, laser sources, etc. A data source produce pseudo random sequence of bit at the rate of $2.5 \mathrm{Gbps}$. The output of this source given to modulator driver [7]. Basically NRZ pulse of the duty cycle 0.5 is produced from modulator driver [8]. Output of source is CW type and its at frequency of $1550 \mathrm{~nm}$ or $193.1 \mathrm{THz}$ and spacing is 0.8 with $\mathrm{P}_{\text {out }}$ is $5 \mathrm{dBm}$

Here, transmission fiber is $180 \mathrm{Km} \mathrm{SMF}$ and DCF is $35 \mathrm{Km}$ long. This adjustment for to give fully compensation for the dispersion slope and accumulated dispersion in the transmission fiber [9]. The total channel length is $215 \mathrm{Km}$ long. Modulator of Mach-Zehnder has 30dB. At the receiver side optical signal transform into electrical signal using pin diode. Generally pin diode have responsivity up to $1 \mathrm{~A} / \mathrm{W}$ and dark current is $10 \mathrm{nA}$. Also here we use optical filter at receiver sides.

Table 1. Simulation Parameters

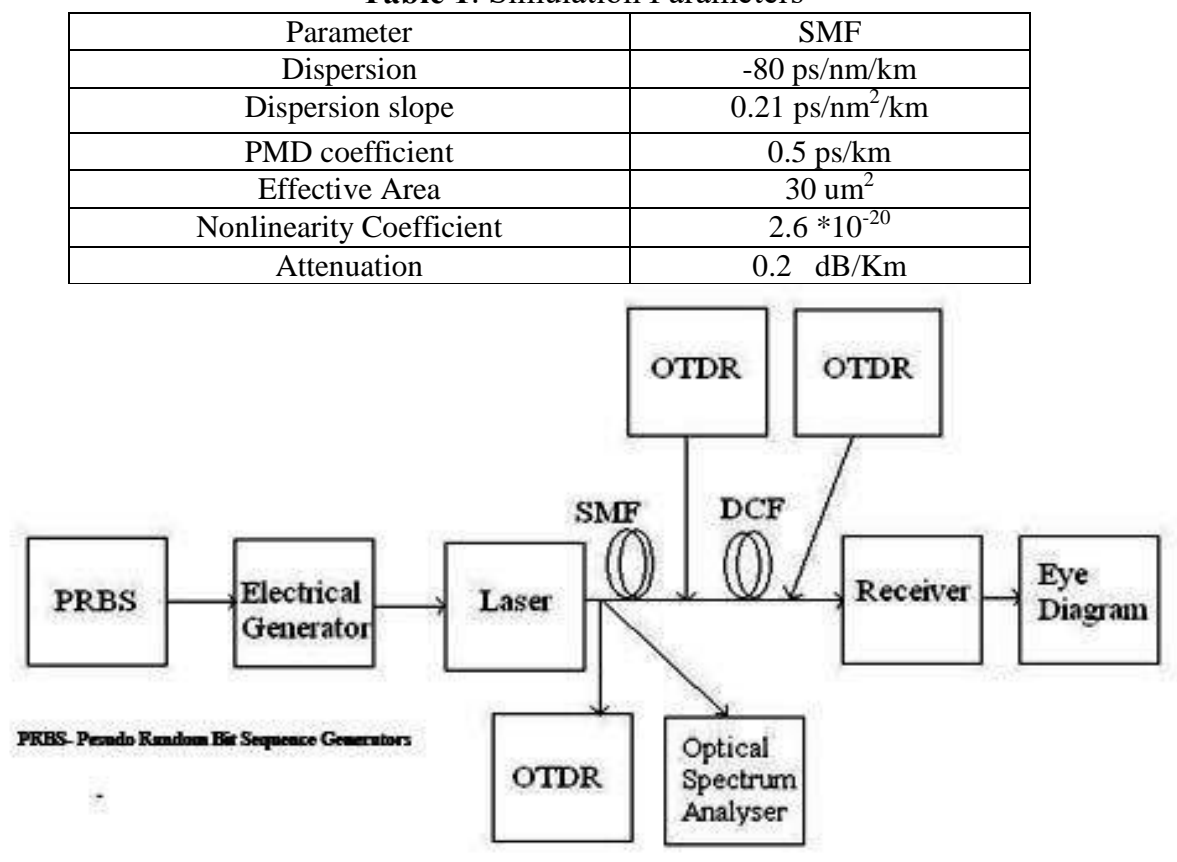

Figure 2. Basic Implementation of System

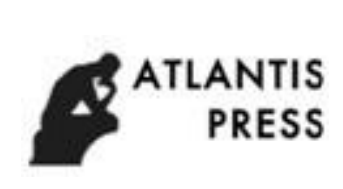




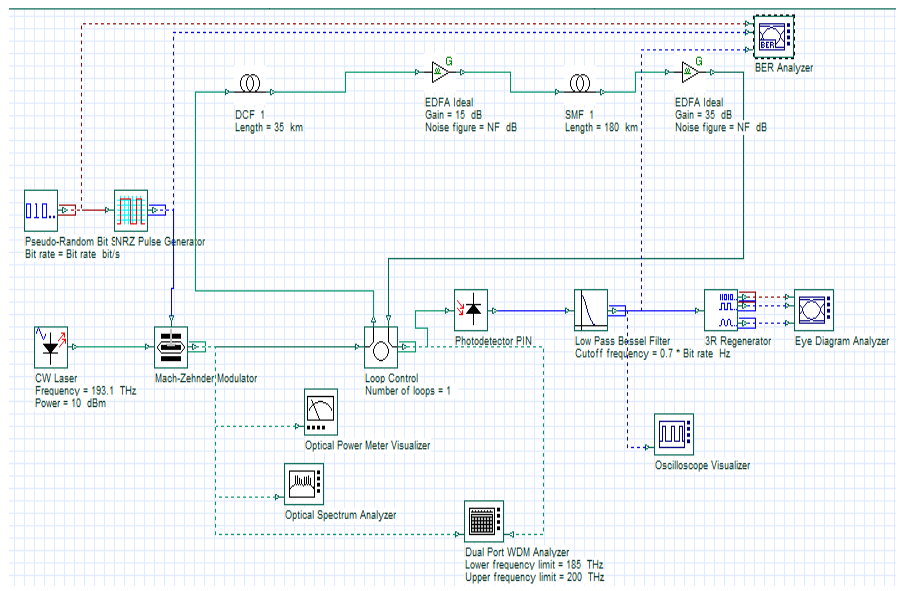

Fig. A. Pre Compensation

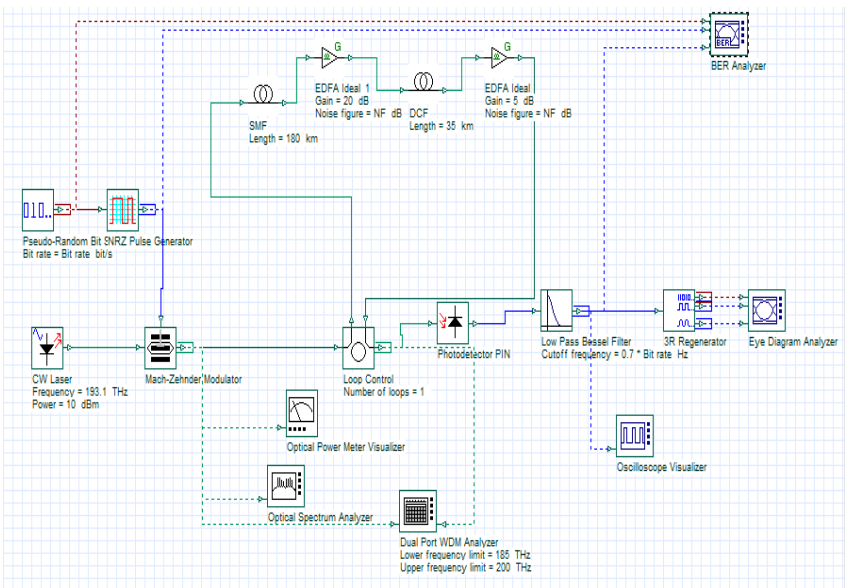

Fig. B. Post Compensation

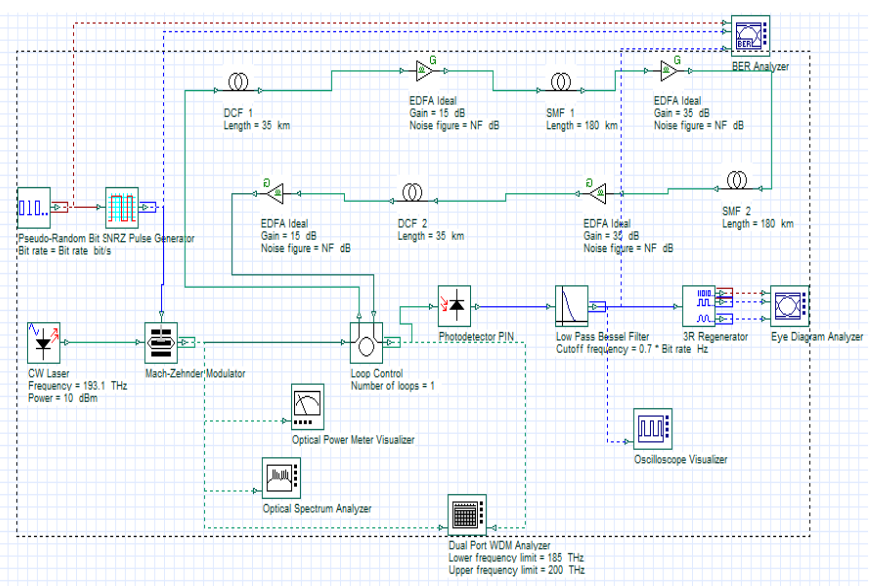

Fig. C. Symmetrical/mix-compensation

Fig. 3. Pre, Post and Symmetrical Compensation scheme

\section{Mathematical Design}

Here we gives mathematical module for DCF.

Bit Rate $=2.5 \mathrm{Gbps}, \quad$ for $1550 \mathrm{~nm}$.; coupling loss $(\mathrm{L})=1 \mathrm{~dB}$; System Margin $(\mathrm{M})=5 \mathrm{~dB}$ 
Transmitted output power $\left(\mathrm{P}_{\mathrm{t}}\right)=0 \mathrm{dBm}$; Receiver sensitivity $(\mathrm{R})=-35 \mathrm{dBm}$

In fig. 3 we see fiber optic system with DCF and SMF to reduction of chromatic dispersion. Dispersion compensated fiber (DCF) provide an optical medium with relatively large chromatic dispersion factor $(D(\lambda))$ at operating wavelength [6].

If length of SMF is $\mathrm{L}_{\mathrm{SMF}}$ connected in series with DCF length $\mathrm{L}_{\mathrm{DCF}}$

Maximum allowable fiber loss $=\mathrm{P}_{\mathrm{t}}-(\mathrm{R}+\mathrm{M}+\mathrm{L})$

So, maximum allowable fiber loss $=0-(-35+1+5)=29$

$$
\begin{aligned}
& \text { Maximum allowable CD }(\mathrm{t})=\frac{1}{(4 \times \text { Bit rate })} \\
& \text { Maximum allowable CD }(\mathrm{t})=\frac{1}{(4 \times 2.5109)}=100 \mathrm{ps}
\end{aligned}
$$

And, Allowable CD $(\mathrm{t})=100 \mathrm{ps}=\mathrm{L}_{\mathrm{SMF}} \mathrm{D}_{\mathrm{SMF}}(\lambda) \Delta \lambda+\mathrm{L}_{\mathrm{DCF}} \mathrm{D}_{\mathrm{DCF}} \Delta \lambda$

Total loss of two fiber combination $=29$ at, $\lambda=1550 \mathrm{~nm}$.

So, for dispersion factor for DCF $=-80 \mathrm{ps} / \mathrm{nm} \cdot \mathrm{km}$

Dispersion factor for $\mathrm{SMF}=16 \mathrm{ps} / \mathrm{nm} . \mathrm{km}$

As solving equation (1) and (2) we get,

$\mathrm{L}_{\mathrm{SMF}}=170 \mathrm{Km}$ and $\mathrm{L}_{\mathrm{DCF}}=9.5 \mathrm{Km}$ approx. $10 \mathrm{Km}$.

\section{Result and analysis}

In optical communication systems, only optical signal to noise ratio (OSNR) could not accurately measure the system performance, especially in WDM systems. Typically, as a quality factor, Q is a one of the important factor to measure the optical system performance by which to characterize the BER [10]. As a Bit error rate is a total number of bit errors per unit time. BER is unit less quantity which measure performance of optical transmission system. as it express in percentage.

For analysis of results we have used eye diagram. An eye diagram is graphical representation of overall performance of optical fiber system. A Eye diagram is actually a voltage diagram that displays a numbers of superimposed data pattern that resemble an eye. In eye diagram basic parameters that are analyzed include Qfactor, BER (bit error rate), Eye height and threshold value. In eye height is also called as vertical eye opening and it measure a noise margin of the system.

Eye height is given by following formula

$$
\text { Eye hight }=\mathrm{P}_{\text {top }}-3 * \mathrm{SD}_{\text {top }}+\mathrm{P}_{\text {base }}-3 * \mathrm{SD}_{\text {base }}
$$

as, $\quad \mathrm{P}_{\text {top }}=$ Mean of most predominant peak of histogram for high logic.

$\mathrm{P}_{\text {base }}=$ Mean of most predominant peak of histogram for Low logic.

$\mathrm{SD}_{\text {top }}=$ Standard devation for high logic.

$\mathrm{SD}_{\text {base }}=$ Standard deviation for low logic .

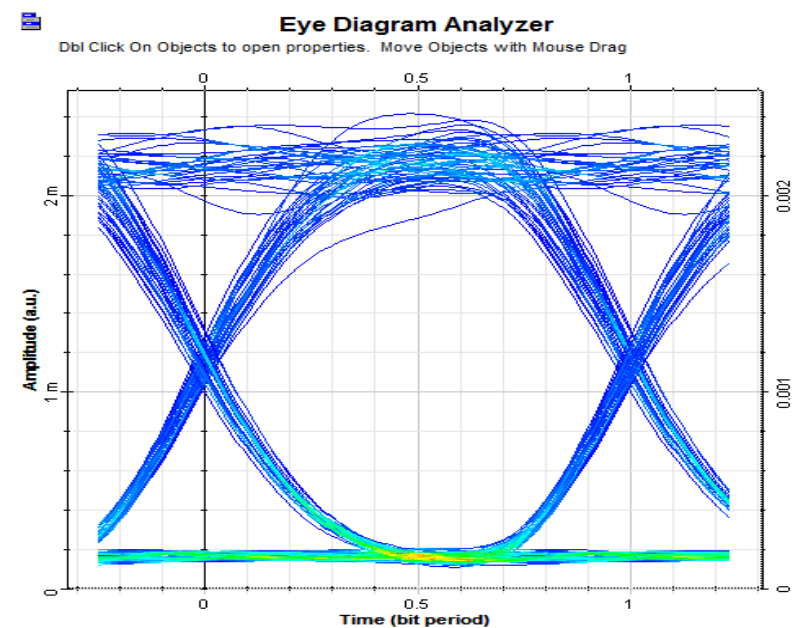

Fig. a) Pre-compensation 


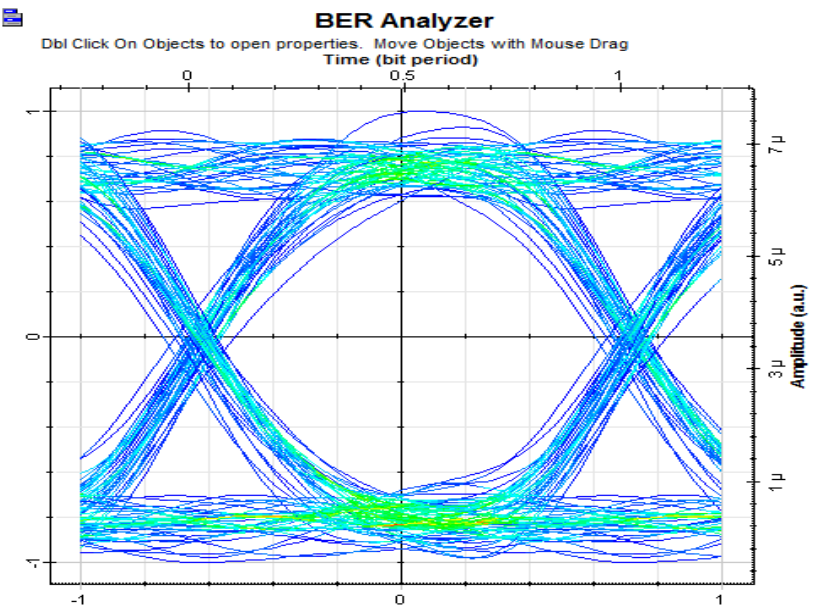

Fig b) Post-compensation

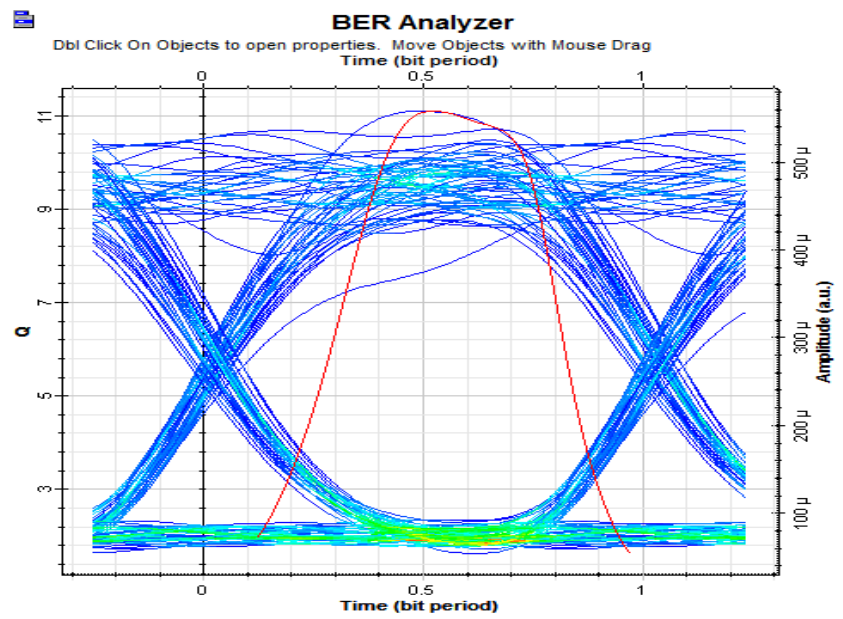

Fig c) Symmetrical/mix-compensation.

Fig. 4. Eye diagrams

Table 2. Comparison of 3 dispersion compensation schemes

\begin{tabular}{cccc}
\hline & Pre -compensation & Post -compensation & $\begin{array}{c}\text { Symmetrical } \\
\text { compensation }\end{array}$ \\
\hline $\begin{array}{c}\text { Q-factor } \\
(\mathrm{db})\end{array}$ & 11.097 & 11.775 & 18.71 \\
BER & $5.162 \mathrm{e}^{-29}$ & $1.513 \mathrm{e}^{-78}$ & $2.5979 \mathrm{e}^{-32}$ \\
Eye height & 0.0002923 & 0.0004651 & 0.0001681 \\
$\begin{array}{c}\text { Threshold } \\
\text { value }\end{array}$ & 0.0001573 & 0.0003340 & 0.0004728 \\
\hline
\end{tabular}

In table 2, we can see that, BER of the symmetrical method is $2.597 \mathrm{e}^{-32}$ means that it is better than other pre and post compensation method, If BER of system is less then, probability of error of bit loss during transmission is less and data transmission for long distance is achieve easily. Also $Q$ factor of symmetrical system is 18.71 on $9.8 \mathrm{~km}$ distance for both practically and mathematically approximately equal so, symmetrical compensation method is much better result than other methods of compensation. 


\section{Conclusion}

In this work namely, Pre, Post and Symmetrical compensation methods of dispersion were analysed for optical fiber communication. Experimental results show that for a single mode fiber (SMF) proposed symmetrical compensation technique is better than Pre and Post compensation techniques in terms of reduced BER and improved quality factor $\mathrm{Q}$ of signal. So we proposed this technique for high data rate and long distance transmission in an optical communication system.

\section{References}

[1] K. Thyagarajan, R. K. Varshney, P. Palai, A. K. Ghatak, and I. C. Goyal : A Novel Design of a Dispersion Compensating Fiber. IEEE Photonics Technology Letters, Vol. 8, No. 11, November 1996.

[2] Laxman Tawade,Shantanu Jagadale,Munir Sayyad,Sanjay Nalbalwar : A Novel Analysis Of Single Mode Fiber For Reduction Of Chromatic Dispersion Using Dispersion Compensated Fiber,Springer, DOI 10.1007/978-3-642,2010.

[3] Manpreet Kaur,Himali Saragal : Dispersion Compensation With Dispersion Compensating Fiber (DCF), DOI 10.17148/IJARCCE.2015.4280.

[4] Rajiv Ramaswami, Kumar N. Sivarajan : Chapter 2. Propagation Of Signal In Optical Fiber, http://dx.doi.org/10.1016/B978-0-12-374092-2.50010-2.

[5] Kaikai Xu,Yang Ou.: Theoretical and numerical characterization of a $40 \mathrm{Gbps}$ long-haul multichannel transmission system with dispersion compensation, Digital Communications and Networks(2015) 1, 222-228,2352-8648/\&2015. Production and hosting by Elsevier B.V. ,http://dx.doi.org/10.1016/j.dcan.2015.06.001.

[6] Gunpreet Kaur,Ranvir Kaur : Use Of Dispersion Compensating Fiber In Optical Transmission Network For Sine Modulator Format, IJISET Vol.1,issue 7,Sept. 2014.

[7] Divya Dhawan,Nina Gupta : Optimization Of Fiber Based Dispersion Compensated In RZ And NRZ Data Modulation Formats, Journals of engineering and science technology, Vol 6,No.6,2011.

[8] Ladislav stepanak : Chromatic dispersion in optical communication, Number 2, Vol VIII, Jul 2012.

[9] Rupinder Kaur, Mandeep Singh : Dispersion Compensation in Optical Fiber Communication System Using WDM with DCF and FBG, IOSR Journal of Electronics and Communication Engineering (IOSR-JECE),e-ISSN: 2278-2834,p- ISSN: 2278-8735.Volume 11, Issue 2, Ver. II (May-Jun .2016), PP 122-130.

[10] Bo-ning HU, Wang Jing, Wang Wei,Rui-mei Zhao : Analysis on Dispersion Compensation with DCF based on Optisystem, 2nd International Conference on Industrial and Information Systems,978-1-4244-8217-7110/2010 IEEE. 